\title{
Robust Fault Detection with Unknown-Input Interval Observers using Zonotopes
}

\author{
P. Guerra* V. Puig * M. Witczak** \\ * Automatic Control Department, Universitat Politècnica de Catalunya \\ (UPC), Rambla de Sant Nebridi, 11, 08222 Terrassa (Spain); (e-mail: \\ vicenc.puig@upc.edu). \\ ** Institute of Control and Computation Engineering, University of Zielona \\ Góra, 50 Podgórna, 65-246 Zielona Góra (Poland), (e-mail: \\ m.witczak@issi.uz.zgora.pl)
}

\begin{abstract}
This paper presents the problem of robust fault detection using unknown-input interval observers. These observers face the robustness problem using two complementary strategies. First, disturbances considered as unknown inputs are decoupled. Second, process/measurement noise and modeling uncertainty are considered unknown but bounded by intervals. Their effect is addressed using an interval state observation method based on zonotope representation of the set of possible states. Finally, an example based on a linearized model of a flight control system is proposed to show the effectiveness of the proposed approach. Copyright (C)2008 IFAC
\end{abstract}

\section{INTRODUCTION}

Model-based fault detection is based on the use of mathematical models of the monitored system. The better the model used to represent the dynamic behavior of the system, the better is the chance of improving the reliability and performance in detecting faults. However, modeling errors and disturbances in complex engineering systems are inevitable, and hence there is a need to develop robust fault detection algorithms. The most common approach to deal with the robustness problem in the Fault Detection and Isolation (FDI) community is based on the decoupling principle. Using this approach, the residual is designed to be insensitive to unknown disturbances, whilst sensitive to faults using the unknown input observer, eigenstructure assignment proposed by Chen and Patton (1999) or structured parity equations proposed by Gertler (1998). However, the robustness problem with respect to modeling errors is more difficult to solve, because its distribution matrix is normally unknown and should be estimated, being many times time varying. Moreover, there might be too many disturbances to be decoupled due to the lack of freedom degrees. An alternative strategy is to consider modeling errors as unknown disturbances, and to propagate and bound their effect on the residual, using for example interval methods as in Puig et al. (2002). This will be the approach followed in this paper to handle modeling uncertainties. On the other hand, process and measurement noises are usually modeled stochastically using restrictive assumptions concerning the distribution law (the typical assumption is a zero mean white noise). However, in many practical situations only bounds on the noise signals are available as in Milanese et al. (1996). This approach is used to describe noise signals in this paper. Unfortunately, the set of states obtained propagating parametric and noise bounded uncertainty may become extremely complex. In the literature several approximating sets and related operations have been proposed to enclose and propagate the set of possible states. In Witczak (2007), a state estimator based on enclosing the set of states by the smallest ellipsoid is proposed using the algorithms proposed by Maksarov and Norton (1996). However, in this approach only additive uncertainty is considered, but not the multiplicative uncertainty introduced by modeling uncertainty located in the parameters. Here, both types of uncertainties are considered as in Rinner and U. Weiss (2004), but there only system trajectories obtained from the uncertain parameter interval vertices are considered, assuming that the monotonicity property holds. In this paper, interval observers based on enclosing the set of states by zonotopes are presented without assuming any monotonicity property and considering the whole set of possible trajectories. The paper is organized as follows: In Section 2, interval observers are introduced altogether with the unknown input decoupling principle to handle unknown disturbances. In Section 3 interval observers are applied to fault detection. And, Section 4 presents the implementation of the fault detection approach using zonotopes. Finally, in Section 5 an application example based on a linearised model of a flight control system is presented to illustrate the effectiveness of the proposed approach.

\section{INTERVAL OBSERVERS}

\subsection{System set-up}

Let us consider the following discrete-time linear system:

$$
\begin{gathered}
\mathbf{x}_{k+1}=\mathbf{A}\left(\theta_{k}\right) \mathbf{x}_{k}+\mathbf{B}\left(\theta_{k}\right) \mathbf{u}_{k}+\mathbf{E} \mathbf{d}_{k}+\mathbf{w}_{k} \\
\mathbf{y}_{k}=\mathbf{C} \mathbf{x}_{k}+\mathbf{v}_{k}
\end{gathered}
$$

where:

- $\mathbf{x}_{k} \in R^{n x}, \mathbf{u}_{k} \in R^{n u}, \mathbf{y}_{k} \in R^{n y}$ and $\mathbf{d}_{k} \in R^{n d}$, are state, input, output and unknown input, vectors of dimension $n x$, $n u, n y$ and $n d$ respectively;

- $\mathbf{v}_{k} \in R^{n v}$ and $\mathbf{w}_{k} \in R^{n w}$ are measurement and process noise of dimension $n v=n y$ and $n w=n x$ respectively; that are considered unknown but bounded, i.e. $\mathbf{v}_{k} \in \mathbb{V}_{k}$, and $\mathbf{w}_{k} \in \mathbb{W}_{k}$, where $\mathbb{V}_{k}$ and $\mathbb{W}_{k}$ are interval boxes:

$\mathbb{V}_{k}=\left\{v_{k} \in R^{n v} \mid \underline{v}_{k} \leq v_{k} \leq \bar{v}_{k}\right\}$,

$\mathbb{W}_{k}=\left\{w_{k} \in R^{n \bar{w}} \mid \underline{w}_{k} \leq w_{k} \leq \bar{w}_{k}\right\}$ 
- $\mathbf{A}\left(\theta_{k}\right)$ and $\mathbf{B}\left(\theta_{k}\right)$ are the state space matrices and $\theta_{k}$ is a vector of uncertain time-varying parameters of dimension $p$ with their values bounded by an interval box ${ }^{1}$ : $\Theta=\left\{\theta \in R^{p} \mid \underline{\theta} \leq \theta \leq \bar{\theta}\right\}$.

- $\mathbf{C}$ is the output equation matrix;

- E stands for the "unknown input" distribution matrix and represents the influence of the unknown disturbances on the system. In this paper as a first approach, it is assumed to be a known matrix. It is let as a future work considering the associated modelling errors, i.e. $\mathbf{E}\left(\theta_{k}\right)$.

\subsection{Decoupling the unknown input}

The effect of unknown inputs can be tackled using several methods, as for example by introducing an additional matrix into the state estimation equation, which is then used for decoupling the unknown inputs effect on the state estimation error according to Chen and Patton (1999), or transforming adequately the system (1) into a system without the unknown input as in Keller (1999). See Hui and Zak (2005) for a more in depth study of design methods for unknown input observers. In any case, the necessary condition for the existence of a solution to the unknown input decoupling problem (See Chen and Patton (1999)):

$$
\operatorname{rank}(\boldsymbol{C E})=\operatorname{rank}(\boldsymbol{E})=n d
$$

If the condition (3) is satisfied, then it is possible to calculate $\mathbf{O}=(\mathbf{C E})^{+}=\left[(\mathbf{C E})^{T} \mathbf{C E}\right]^{-1}(\mathbf{C E})^{T}$, where $(\cdot)^{+}$stands for the pseudoinverse of its argument. Then, by multiplying (2) by $\mathbf{O}$, it is straightforward to show that

$$
\mathbf{d}_{k}=\mathbf{O}\left[\mathbf{y}_{k+1}-\mathbf{C}\left(\mathbf{A}\left(\theta_{\mathbf{k}}\right) \mathbf{x}_{\mathbf{k}}+\mathbf{B}\left(\theta_{\mathbf{k}}\right) \mathbf{u}_{\mathbf{k}}+\mathbf{w}_{\mathbf{k}}\right)-\mathbf{v}_{\mathbf{k}+\mathbf{1}}\right]
$$

Substituting (4) into (1) gives

$$
\mathbf{x}_{k+1}=\overline{\mathbf{A}}\left(\theta_{k}\right) \mathbf{x}_{k}+\overline{\mathbf{B}}\left(\theta_{k}\right) \mathbf{u}_{k}+\overline{\mathbf{E}} \mathbf{y}_{k+1}+\overline{\mathbf{w}}_{k}
$$

where:

$$
\begin{gathered}
\overline{\mathbf{A}}\left(\theta_{k}\right)=(\mathbf{I}-\mathbf{E O C}) \mathbf{A}\left(\theta_{k}\right) \\
\overline{\mathbf{B}}\left(\theta_{k}\right)=(\mathbf{I}-\mathbf{E O C}) \mathbf{B}\left(\theta_{k}\right), \quad \overline{\mathbf{E}}=\mathbf{E O} \\
\overline{\mathbf{w}}_{k}=(\mathbf{I}-\mathbf{E O C}) \mathbf{w}_{k}-\overline{\mathbf{E}}_{\mathbf{v}_{k+1}}
\end{gathered}
$$

\subsection{The interval observation principle}

Let the model for the state estimator of the system given by (5), once the unknown input is decoupled, be a Luenberger observer formulated as

$$
\begin{aligned}
\hat{\mathbf{x}}_{k+1}= & \overline{\mathbf{A}}\left(\theta_{\mathbf{k}}\right) \hat{\mathbf{x}}_{k}+\overline{\mathbf{B}}\left(\theta_{\mathbf{k}}\right) \mathbf{u}_{k}+\overline{\mathbf{E}} \mathbf{y}_{k+1}+\overline{\mathbf{w}}_{k} \\
& +\mathbf{K}\left(\mathbf{y}_{k}-\hat{\mathbf{y}}_{k}\right) \\
\hat{\mathbf{y}}_{k}=\mathbf{C} \hat{\mathbf{x}}_{k}+\mathbf{v}_{k} &
\end{aligned}
$$

where $\mathbf{K}$ is the observer gain that has to be designed in order to stabilize the observer given by Eq. (6) for all $\theta_{k} \in \Theta$.

Definition 1. Consider the state estimator given by Eq. (6), an initial compact set $\mathbb{X}_{0}$ and a sequence of measured inputs

\footnotetext{
1 This type of model is known, in the literature, as interval model. See, for example, Puig et al. (2002) or Rinner and U. Weiss (2004)
}

$\left(\mathbf{u}_{j}\right)_{0}^{k-1}$ and outputs $\left(\mathbf{y}_{j}\right)_{0}^{k}$. The exact uncertain estimated state set at time $k$ is expressed by

$$
\begin{array}{r}
\mathbb{X}_{k}=\left\{\hat{\mathbf{x}}_{k}:\left(\hat{\mathbf{x}}_{j}=\overline{\mathbf{A}}\left(\theta_{j-1}\right) \hat{\mathbf{x}}_{j-1}+\overline{\mathbf{B}}\left(\theta_{j-1}\right) \mathbf{u}_{j-1}\right.\right. \\
\left.+\overline{\mathbf{w}}_{j-1}+\overline{\mathbf{E}}_{j}+\mathbf{K}\left(\mathbf{y}_{j-1}-\hat{\mathbf{y}}_{j-1}\right)\right)_{j=1}^{k}, \\
\left(\hat{\mathbf{y}}_{j-1}=\mathbf{C}_{j-1}+\mathbf{v}_{j-1}\right)_{j=1}^{k} \quad \mathbf{x}_{0}, \hat{\mathbf{x}}_{0} \in \mathbb{X}_{0}, \\
\left.\left(\theta_{j-1} \in \Theta, \overline{\mathbf{w}}_{j-1} \in \mathbb{W}_{j-1}, \mathbf{v}_{j-1} \in \mathbb{V}_{j-1}\right)_{j=1}^{k}\right\}
\end{array}
$$

The uncertain state set described in Definition 1 at time $k$ can be computed approximately by admitting the rupture of the existing relations between variables of consecutive time instants. ${ }^{2}$ This makes possible to compute an approximation of this set from the approximate uncertain state set at time $k-1$. Because the exact set of estimated states would be difficult to compute, one straightforward way to bound this set is using a box (interval hull) as in Puig et al. (2002), a zonotope as in Alamo et al. (2005), an ellipsoid as in Maksarov and Norton (1996) or other geometric regions easy to compute. Before introducing such algorithm an additional definition is introduced.

Definition 2. Consider a system given by Eq. (6), the set of uncertain states at time $k-1, \mathbb{X}_{k-1}$ and the input/ouput values $\left(\mathbf{u}_{k-1}, \mathbf{y}_{k-1}, \mathbf{y}_{k}\right)$. Then, the approximated set of estimated states at time $k$ based on the measurements up to time $k-1$ is defined as

$$
\begin{array}{r}
\mathbb{X}_{k}^{e}=\left\{\hat{\mathbf{x}}_{k}: \overline{\mathbf{A}}\left(\theta_{k-1}\right) \hat{\mathbf{x}}_{k-1}+\overline{\mathbf{B}}\left(\theta_{k-1}\right) \mathbf{u}_{k-1}+\overline{\mathbf{w}}_{k-1}\right. \\
+\overline{\mathbf{E}} \mathbf{y}_{k}+\mathbf{K}\left(\mathbf{y}_{k-1}-\hat{\mathbf{y}}_{k-1}\right), \quad \hat{\mathbf{y}}_{k-1}=\mathbf{C} \hat{\mathbf{x}}_{k-1}+\mathbf{v}_{k-1} \\
\left.\hat{\mathbf{x}}_{k-1} \in \mathbb{X}_{k-1}, \theta_{k-1} \in \Theta, \overline{\mathbf{w}}_{k-1} \in \mathbb{W}_{k-1}, \mathbf{v}_{k-1} \in \mathbb{V}_{k-1}\right\}
\end{array}
$$

Analogously, considering measurement equation in Eq. (6) the approximated set of estimated outputs $\mathbb{Y}_{k}^{e}$ can be determined.

Using previous definition, the set of estimated states (or outputs) introduced in Definition 1 will be approximated iteratively using zonotopes. From these zonotopes, an interval for each state variable can also be obtained by computing the interval hull of the zonotope. The sequence of interval hulls $\square \mathbb{X} e_{k}^{e}$ with $k \in[0, N]$ will be called the interval observer estimation of the system given by Eq. (6). Analogously, the sequence of interval hulls $\square \mathbb{Y}_{k}^{e}$ can be obtained. Following previous idea, Algortihm 1 is proposed to determine an approximation of set of uncertain estimated states.

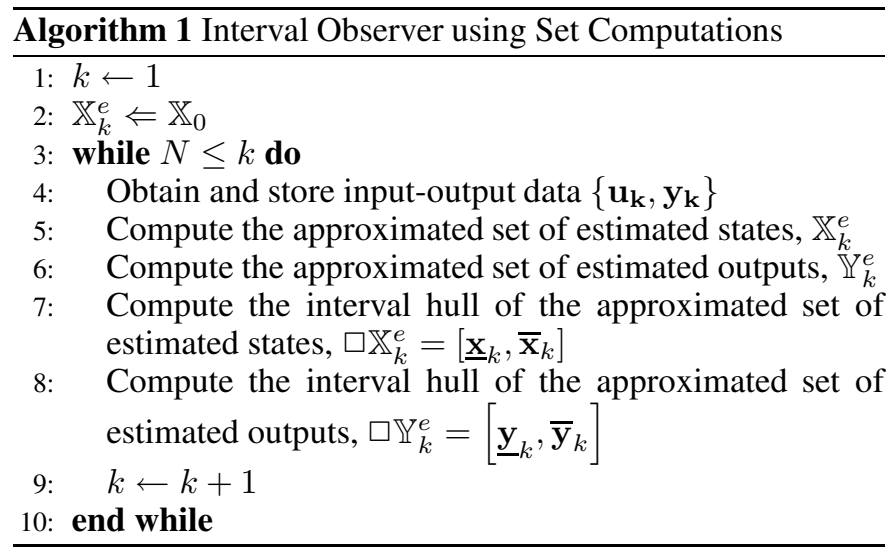

2 However, the problem of uncertainty propagation (wrapping effect) could appear when this set is approximated in this way because of the accumulation of overestimation along the simulation time and deriving in an explosion of uncertainty. 


\section{FAULT DETECTION USING INTERVAL OBSERVERS}

The application of observers to fault detection consists in testing whether the measured output is consistent with the one by an observer using a faultless model. If an inconsistency is detected, the existence of a fault is proved. In the case of assuming bounded noise, the measurement can be considered to be in the interval $\left[\mathbf{y}_{\mathbf{k}}\right]$. Then, the fault detection test can be stated as

$$
\left[\mathbf{y}_{\mathbf{k}}\right] \cap \mathbb{Y}_{k}^{e} \neq \emptyset
$$

where $\mathbb{Y}_{k}^{e}$ is set of predicted outputs that can be obtained using Algorithm 1.

Algorithm 2 implements fault detection using interval observers and the fault detection test presented in (9).

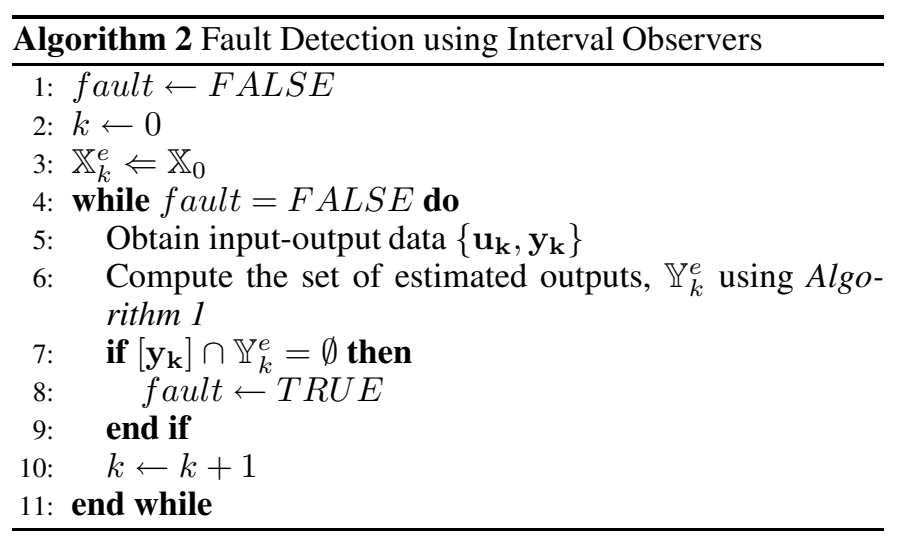

\section{IMPLEMENTATION USING ZONOTOPES}

\subsection{Introduction}

In this paper, zonotopes are used to bound the set of uncertain estimated sets. Let us introduce zonotopes.

Definition 3. The Minkowski sum of two sets $\mathbb{X}$ and $\mathbb{Y}$ is defined by $\mathbb{X} \oplus \mathbb{Y}=\{\mathbf{x}+\mathbf{y}: \mathbf{x} \in \mathbb{X}, \mathbf{y} \in \mathbb{Y}\}$.

Definition 4. Given a center vector $\mathbf{p} \in \mathbb{R}^{n}$ and a matrix $\mathbf{H}$ $\in \mathbb{R}^{n \times m}$ the Minkowski sum of the segments defined by the columns of matrix $\mathbf{H}$, is called a zonotope of order m. This set is represented as:

$$
\mathbb{X}=\mathbf{p} \oplus \mathbf{H B}^{m}=\left\{\mathbf{p}+\mathbf{H z}: \mathbf{z} \in \mathbf{B}^{m}\right\}
$$

where: $\mathbf{B}^{m}$ is a unitary box, composed by $\mathrm{m}$ unitary intervals.

Then, a zonotope $\mathbb{X}$ of order $m$ can be viewed as the Minkowski sum of $m$ segments. The order $m$ is a measure for the geometrical complexity of the zonotopes.

Definition 5. The interval hull $\square \mathbb{X}$ of a closed set $\mathbb{X}$ is the smallest interval box that contains $\mathbb{X}$.

Given a zonotope $\mathbb{X}=\mathbf{p} \oplus \mathbf{H B}^{m}$, its interval hull can be easily computed by evaluating $\mathbf{p} \oplus \mathbf{H B}^{m}$, for all $i=1 . . n$ : $\square \mathbb{X}=\left\{\mathbf{x}:\left|\mathbf{x}_{\mathbf{i}}-\mathbf{p}_{\mathbf{i}}\right| \leq\left\|\mathbf{H}_{\mathbf{i}}\right\|_{\mathbf{1}}\right\}$ where $\mathbf{H}_{i}$ is $i^{t h}$-row of $\mathbf{H}$, and $\mathbf{x}_{\mathbf{i}}$ and $\mathbf{p}_{\mathbf{i}}$ are $i^{\text {th }}$ components of $\mathbf{x}$ and $\mathbf{p}$, respectively.

\subsection{Implementation of interval observers using zonotopes}

To implement interval observers using zonotopes, it should be noticed that using Eq. (6) as the expression of the estimator model, it can be viewed as a discrete-time system with one input that can be reorganized as:

$$
\hat{\mathbf{x}}_{k+1}=\overline{\mathbf{A}}\left(\theta_{\mathbf{k}}\right)_{o} \hat{\mathbf{x}}_{k}+\overline{\mathbf{B}}_{o}\left(\theta_{k}\right) \mathbf{u}_{k}^{o}
$$

where:

$\overline{\mathbf{A}}_{o}=\overline{\mathbf{A}}\left(\theta_{\mathbf{k}}\right)-\mathbf{K C}, \overline{\mathbf{B}}_{o}=\left[\overline{\mathbf{B}}\left(\theta_{\mathbf{k}}\right) \overline{\mathbf{E}} \mathbf{I} \mathbf{K} \mathbf{K}\right]$ and $\mathbf{u}_{k}^{0}=$ $\left[\begin{array}{lllll}\mathbf{u}_{k} & \mathbf{y}_{k+1} & \overline{\mathbf{w}}_{k} & \mathbf{y}_{k} & \mathbf{v}_{k}\end{array}\right]^{t}$.

Then, the problem of interval observation can be formulated as a problem of interval simulation and requires characterizing the set $\mathbb{X}_{k}^{e}$. This set can be viewed as the direct image evaluation of Eq. (10) and can be implemented using zonotopes.

According to Algorithm 1, interval observers involves a bounding operation applied to the set of estimated states $\mathbb{X}_{k}^{e}$.

\subsection{Implementation of prediction set step}

The prediction set step requires characterizing the set $\mathbb{X}_{k}^{e}$. This set can be viewed as the direct image evaluation of $\mathbf{f}\left(\mathbf{x}_{k}, \theta_{k}, \overline{\mathbf{w}}_{k}\right)=\overline{\mathbf{A}}_{\mathbf{o}}\left(\theta_{k}\right) \hat{\mathbf{x}}_{k}+\overline{\mathbf{B}}\left(\theta_{k}\right) \mathbf{u}_{k}^{o}+\overline{\mathbf{w}}_{k}$. There are different algorithms to bound such an image using ellipsoids (see Maksarov and Norton (1996)) or zonotopes (see Kühn (1998)). To bound such image using zonotopes the following result is used:

Theorem 1. "Zonotope Inclusion" (see Alamo et al. (2005)). Consider a family of zonotopes represented by $\mathbb{X}=\mathbf{p} \oplus \mathbf{M B}^{m}$ where $\mathbf{p} \in \mathbb{R}^{n}$ is a real vector and $\mathbf{M} \in \mathbb{I}^{n \times m}$ is an interval matrix. A zonotope inclusion $\diamond(\mathbb{X})$ is defined by:

$$
\diamond(\mathbb{X})=\mathbf{p} \oplus[\operatorname{mid}(\mathbf{M} \mathbf{G})]\left[\begin{array}{l}
\mathbf{B}^{m} \\
\mathbf{B}^{n}
\end{array}\right]=\mathbf{p} \oplus \mathbf{J B}^{n+m}
$$

where $\mathbf{G} \in \mathbb{R}^{n \times n}$ is a diagonal matrix that satisfies: $G_{i i}=$ $\sum_{j=1}^{m} \frac{\operatorname{diam}\left(\mathbf{M}_{\mathbf{i j}}\right)}{2}, i=1,2 \ldots n$. with mid denotes the center and diam the diameter of the interval according to Moore (1966). Under this definition, $\mathbb{X} \subseteq \diamond(\mathbb{X})$.

This prediction step aims at computing the zonotope $\mathbb{X}_{k+1}^{e}$ that bounds the trajectory of the system at instant $k+1$, from the previous approximating zonotope at time instant $k, \mathbb{X}_{k}$, using the natural interval extension of Eq. (10) as suggested by Moore (1966) and the zonotope inclusion operator, as a generalization of Kühn's method (see Kühn (1998)):

$$
\mathbb{X}_{k+1}^{e}=\mathbf{p}_{k+1} \oplus \mathbf{H}_{k+1} \mathbf{B}^{r}
$$

where:

$$
\mathbf{p}_{k+1}=\operatorname{mid}\left(\overline{\mathbf{A}}_{\mathbf{o}}\left(\theta_{k}\right)\right) \mathbf{p}_{k}+\operatorname{mid}\left(\overline{\mathbf{B}}_{\mathbf{o}}\left(\theta_{k}\right)\right) \mathbf{u}_{\mathbf{k}}^{\mathbf{o}}
$$

and

$$
\begin{aligned}
& \mathbf{H}_{k+1}=\left[\begin{array}{lll}
\mathbf{J}_{\mathbf{1}} & \mathbf{J}_{\mathbf{2}} & \mathbf{J}_{\mathbf{3}}
\end{array}\right] \\
& \mathbf{J}_{\mathbf{1}}=\diamond\left(\overline{\mathbf{A}}_{\mathbf{o}}\left(\theta_{\mathbf{k}}\right) \mathbf{H}_{k}\right) \\
& \mathbf{J}_{\mathbf{2}}=\mathbf{p}_{\mathbf{k}}\left(\operatorname{diam}\left(\overline{\mathbf{A}}_{\mathbf{o}}\left(\theta_{k}\right)\right) / 2\right) \\
& \mathbf{J}_{\mathbf{3}}=\mathbf{u}_{\mathbf{k}}^{\mathbf{o}}\left(\operatorname{diam}\left(\overline{\mathbf{B}}_{\mathbf{o}}\left(\theta_{k}\right)\right) / 2\right)
\end{aligned}
$$

$\mathbf{J}_{\mathbf{1}}$ is calculated using the zonotope inclusion operator.

It is important to notice that the set of estimated states has an increasing number of segments generating the zonotope $\mathbb{X}_{k+1}^{e}$ using this method. In order to control the domain complexity, a reduction step is thus implemented. Here we use the method proposed in Combastel (2003) to reduce the zonotope complexity.

\subsection{Checking for intersection emptiness}

The step 6 of Algorithm 2 requires to check if the intersection of $\left[\mathbf{y}_{\mathbf{k}}\right] \cap \mathbb{Y}_{k}^{e}$, is not the empty set, before introducing such 
operation, an additional definition is introduced.

Definition 6. Given the zonotope $\mathbb{Y}_{k}^{e}=\mathbf{p} \oplus \mathbf{H B}^{r}$, the strip $\left[\mathbf{y}_{\mathbf{k}}\right]=\left\{\mathbf{x} \in \mathbb{R}^{\mathbf{n}}\left|\mathbf{c}^{\mathbf{T}} \mathbf{x}-d\right| \leq \sigma\right\}$, a hyperplane $S=\{x:$ $\left.\mathbf{c}^{\mathbf{T}} x=q\right\}$ is a supporting hyperplane of a zonotope $\mathbb{Y}_{k}^{e}$ if either $\mathbf{c}^{\mathbf{T}} \mathbf{x} \leq q, \forall \mathbf{x} \in \mathbb{Y}_{k}^{e}$ or else $\mathbf{c}^{\mathbf{T}} \mathbf{x} \geq q, \forall \mathbf{x} \in \mathbb{Y}_{k}^{e}$ with equality occurring for some $\mathbf{x} \in \mathbb{Y}_{k}^{e}$. The two constants $q_{u}$ and $q_{d}$ characterizing the supporting hyperplanes are easily calculated as:

$$
\begin{aligned}
& q_{u}=\mathbf{c}^{\mathbf{T}} \mathbf{p}+\left\|\mathbf{H}^{\mathbf{T}} \mathbf{c}\right\|_{\mathbf{1}} \\
& q_{d}=\mathbf{c}^{\mathbf{T}} \mathbf{p}-\left\|\mathbf{H}^{\mathbf{T}} \mathbf{c}\right\|_{\mathbf{1}}
\end{aligned}
$$

where $\|\cdot\|_{1}$ is the 1 -norm of a vector. Then the intersection check is very easy to perform considering that each new measurement defines a set of consistent states defined by

$$
F_{k}=\left\{\mathbf{x}_{k} \in \mathbb{R}^{n}:-\sigma \leq \mathbf{y}_{\mathbf{k}}-\mathbf{C x}_{\mathbf{k}} \leq \sigma\right\}
$$

where $F_{k}$ is the region between two hyperplanes and the output $y_{k}$ is considered component-wise. The normalized form of this strip is written as

$$
\bar{F}_{k}=\left\{\mathbf{x}_{k} \in \mathbb{R}^{n}:\left|\frac{\mathbf{y}_{\mathbf{k}}}{\sigma}-\mathbf{c}^{\mathbf{T}} \mathbf{x}_{\mathbf{k}}\right| \leq \mathbf{1}\right\}
$$

Calculating the supporting hyperplane constant $q_{u}$ and $q_{d}$ the intersection is empty if and only if:

$$
q_{u}<\frac{\mathbf{y}_{\mathbf{k}}}{\sigma}-1 \text { or } q_{d}>\frac{\mathbf{y}_{k}}{\sigma}+1
$$

This condition of inconsistency for a SISO model was reported in Vicino and Zappa (1996).

\section{CASE STUDY}

\subsection{Description}

A modified version of the benchmark problem proposed in Chen and Patton (1996) is considered. It consists of a linearized discrete-time model of a simplified longitudinal flight control system given by:

$$
\begin{gathered}
\mathbf{x}_{k+1}=\mathbf{A}\left(\theta_{k}\right) \mathbf{x}_{k}+\mathbf{B}\left(\theta_{k}\right) \mathbf{u}_{k}+\mathbf{E} \mathbf{d}_{k}+\mathbf{w}_{k} \\
\mathbf{y}_{k}=\mathbf{C}_{k} \mathbf{x}_{k}+\mathbf{v}_{k}
\end{gathered}
$$

with:

$$
\begin{aligned}
& \mathbf{A}\left(\theta_{k}\right)=\left[\begin{array}{ccc}
a_{11} \pm \Delta a_{11} & a_{12} \pm \Delta a_{12} & a_{13} \pm \Delta a_{13} \\
a_{21} \pm \Delta a_{21} & a_{22} \pm \Delta a_{22} & a_{23} \pm \Delta a_{23} \\
0 & 0.8187 & 0
\end{array}\right] \\
& \mathbf{B}\left(\theta_{k}\right)=\left[\begin{array}{c}
b_{1} \pm \Delta b_{1} \\
b_{2} \pm \Delta b_{2} \\
0.1813
\end{array}\right], \quad \mathbf{C}_{k}=\mathbf{I}_{3 x 3}, \quad \mathbf{E}_{k}=\left[\begin{array}{ll}
0 & 0 \\
1 & 0 \\
0 & 1
\end{array}\right]
\end{aligned}
$$

where: $a_{11}=0.994 \pm \Delta a_{11}, a_{12}=-0.120 \pm \Delta a_{12}, a_{13}=$ $-0.430 \pm \Delta a_{13}, a_{21}=0.002 \pm \Delta a_{21}, a_{22}=0.990 \pm$ $\Delta a_{22}, a_{23}=-0.074 \pm \Delta a_{23}, b_{1}=0.4252 \pm \Delta b_{1}$ and $b_{2}=$ $-0.0082 \pm \Delta b_{2}$

The states variables $\mathbf{x}=\left[\eta_{y} \omega_{z} \delta_{z}\right]^{T}$ represent the normal velocity, pitch rate and pitch angle, respectively. The control input is an elevator control signal. The system has been simulated using $\mathbf{u}_{k}=10$. The covariance matrices for process and measurement noise sequences are $\mathbf{Q}_{k}=\operatorname{diag}\left\{0.1^{2}, 0.1^{2}, 0.01^{2}\right\}$ and $\mathbf{R}_{k}=0.01^{2} I_{3 \times 3}$. The process $\mathbf{w}_{k}$ and measurement $\mathbf{v}_{k}$ noises are normally distributed. The aerodynamic coefficients are randomly perturbed by $\pm 20 \%$, i.e., $\Delta a_{i j}=-0.2 a_{i j}$. The initial state vector used in the simulation was $\mathbf{x}_{o}=\left[\begin{array}{lll}0 & 0 & 0\end{array}\right]^{T}$. The unknown input vector is $\mathbf{d}_{k}=\left[\begin{array}{ll}d_{1} & d_{2}\end{array}\right]^{T}$, where $d_{1}$ and $\mathrm{d}_{2}$ are pulse trains of aperiodic rectangles.
To implement the interval observer based on zonotopes, process/measurement noise only the bound are required (see Eq. (1)). Then, since statistical distribution of noise is given, these bounds will be obtained from the covariances matrices taking 3 times the standard deviation: $\mathbf{w}_{k}=[[-0.3,0.3],[-0.3,0.3]$, $[-0.03,0.03]]$ and $\mathbf{v}_{k}=[[-0.03,0.03],[-0.03,0.03],[-0.03$, $0.03]$. On the other hand, parameter uncertainty is obtained by considering that all parameter values in $\mathbf{A}$ matrix are intervals whose center is the nominal value and the radius is $\Delta a_{i j}=-0.2 a_{i j}$. The gain $\mathbf{K}$ of the interval observer estimator will be designed taking into account statistical distribution of noise. In particular, observer gain $\mathbf{K}$ is determined using covariance matrices for process and measurement noise, $\mathbf{Q}$ and $\mathbf{R}$ respectively, and making use of Kalman filter theory as in Chen and Patton (1996) using steady state approximation:

$$
\mathbf{K}=\left[\begin{array}{ccc}
0.9899 & -0.1203 & -0.4302 \\
0 & 0.9901 & -0.0747 \\
0 & 0.0079 & 0
\end{array}\right]
$$

Then, an optimal Luenberger observer is built using this gain and the system model (17). The interval observer based fault detection method in this application example is initialized using as initial zonotope $\mathbb{X}_{o}$ with: $\mathbf{p}_{o}=\left[\begin{array}{lll}0.1 & 0.1 & 0.1\end{array}\right]^{T}$ and $\mathbf{H}_{o}=$ $I_{3 \times 3}$ and the zonotope order $m$ is limited to 27 .

\subsection{Decoupling the unknown inputs}

Figure 1 shows the three components of the output measurements and their envelopes (+ marks) obtained with the approach presented in this paper without decoupling the unknown inputs and considering a faultless scenario using Algorithm 2. Notice that two components of the output measurement are outside of their envelopes in several time instants. This fact leads to indicate the presence of a fault even in its absence, see Figure 2. This figure shows the fault indication obtained in the faultless scenario without decoupling the unknown inputs: value 0 means no fault while 1 means fault. Using the method described in Section 2, the effect of the unknown input $\mathbf{d}_{k}$ is decoupled. Figure 3 shows the three components of the output measurements and their envelopes obtained with the method after decoupling the unknown inputs and considering a faultless scenario. All components of the output measurement are inside their envelopes at every time instants. Therefore, in this case no fault is indicated. Figure 4 shows the fault signal obtained in the faultless scenario after decoupling the unknown inputs. Since all fault signals are 0 , no fault is indicated. This proves the effectiveness of the method for decoupling the effect of the unknown inputs presented in this paper.

\subsection{Fault detection}

After decoupling the unknown inputs, using the method described in Section 2.2, two different types of fault were studied to evaluate the behavior of the fault detection approach proposed following Algorithm 2: an additive fault and a multiplicative fault.

Additive fault. In this scenario, an additive fault of size 0.5 is introduced in the pitch angle output measurement, i.e. $\mathbf{y}_{\mathbf{k}, \mathbf{3}}=\mathbf{y}_{\mathbf{k}, \mathbf{3}}+0.5$ from time instant $k=10$. Figure 5 shows the three components of the output measurements and their envelopes obtained with the interval observed method based on 


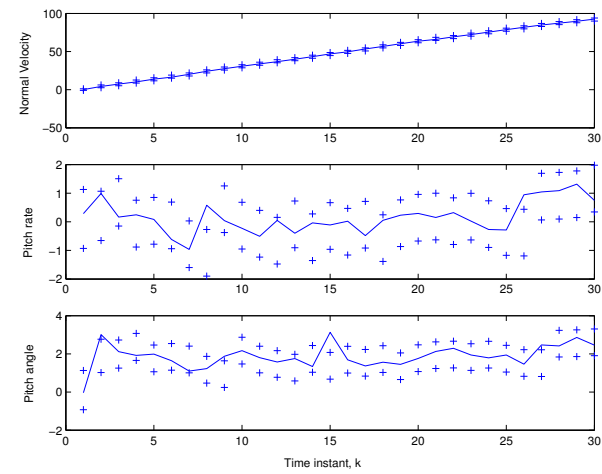

Fig. 1. System output measurements and envelopes (faultless scenario) without decoupling the unknown inputs
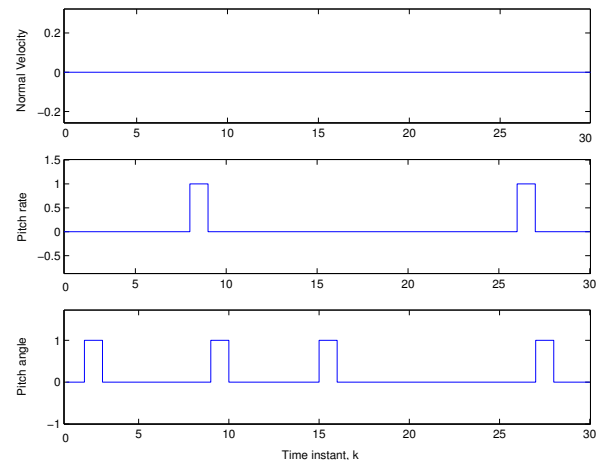

Fig. 2. Faults detected without decoupling the unknown inputs in a faultless scenario
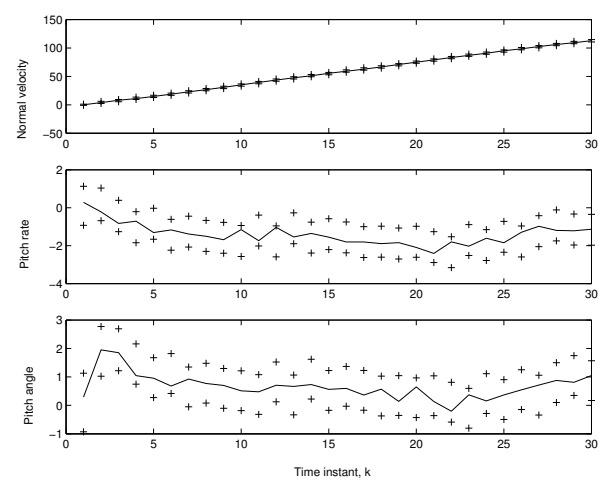

Fig. 3. System output measurements and envelopes (faultless scenario) after decoupling the unknown inputs
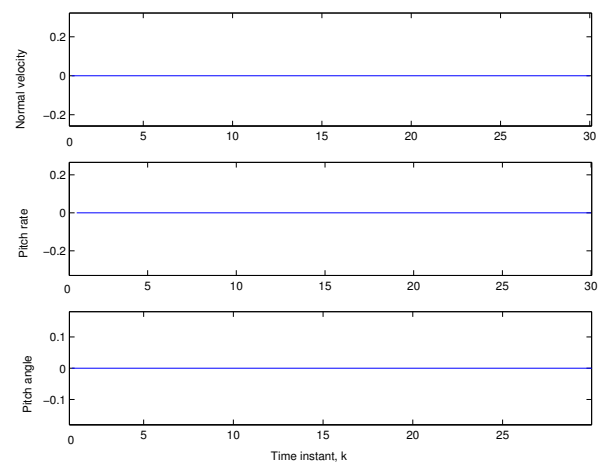

Fig. 4. Fault detection after decoupling the unknown inputs in a faultless scenario zonotopes. Even though the first two components of the output measurement are inside their envelopes, the third component (the pitch angle) goes outside the envelopes for several time instants from $k=10$. Thus, fault is detected. Figure 6 shows the fault signals obtained in this case. In Figure 7, the projection of the approximated set of estimated outputs $\mathbb{Y}_{10}^{e}$ in two components and the measurement output interval considering the measurement noise at time instant $k=10$ are shown. Since these two sets do not intersect, according to the step 7 of Algorithm 2 a fault is indicated.

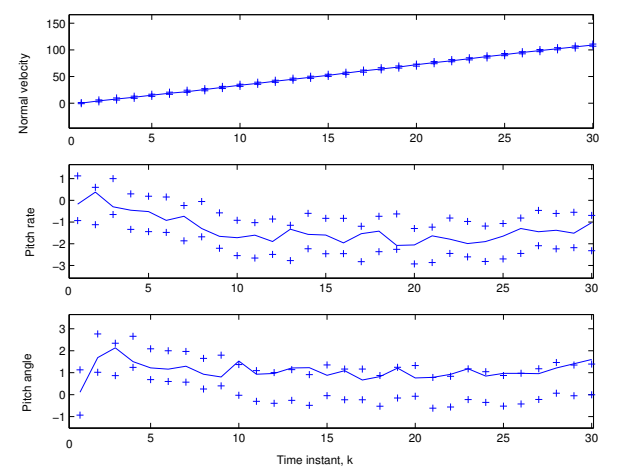

Fig. 5. System output measurements and envelopes (additive fault)
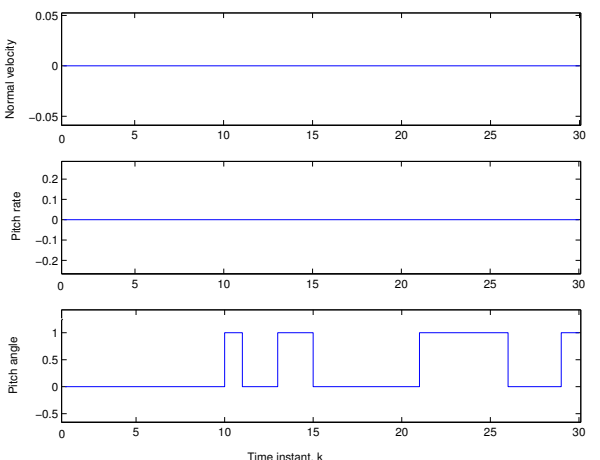

Fig. 6. Interval observer fault detection for an additive fault

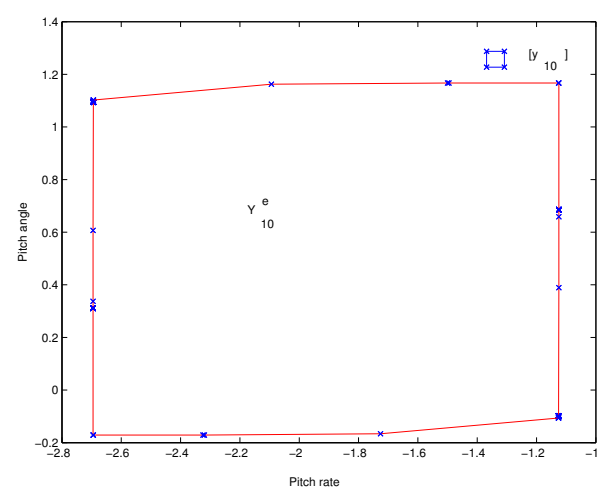

Fig. 7. Approximated set of estimated output and measurement output with noise (additive fault)

Multiplicative fault. In this scenario, a multiplicative fault of size 0.2 is introduced by modifying the parameter $a_{23}$ of the system matrix $\mathbf{A}_{k}$ from time instant $k=10$ as follows: $a_{23}+0.2$. Figure 8 shows the evolution of the three output 
measurements and their envelopes obtained with the interval observer fault detection test based on zonotopes presented in this paper. In this case, all measurements go outside of their envelopes in different time instant since $k=10$, so fault is indicated. Figure 9 shows the fault signals obtained for each component of the measurement outputs. Faults are indicated in all fault signal components. In Figure 10, the projection of the approximated set of estimated outputs and the measurement outputs for pitch rate and pitch angle are presented for time instant $k=10$. Again as these two sets do not intersect, a fault is indicated.
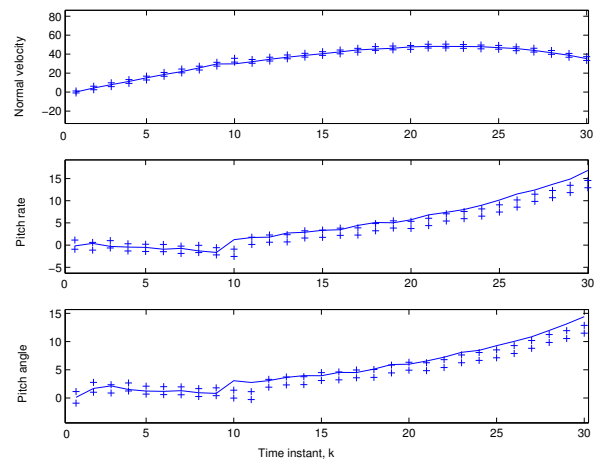

Fig. 8. System output measurements and envelopes (multiplicative fault)
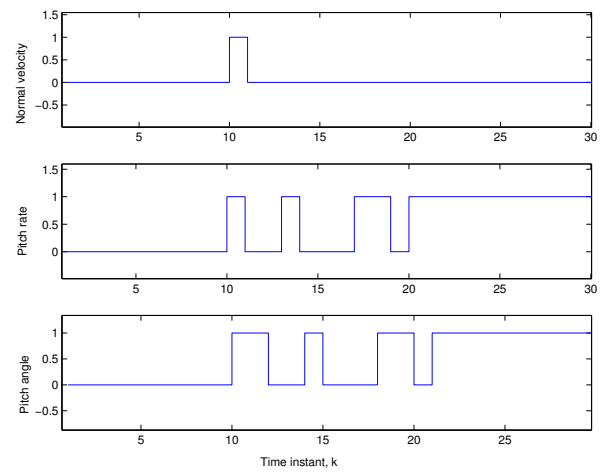

Fig. 9. Interval observer fault detection for a multiplicative fault

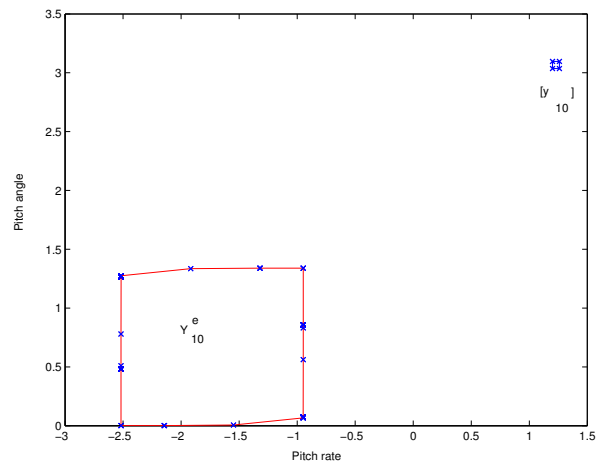

Fig. 10. Approximated set of estimated output and measurement output with noise (multiplicative fault)

\section{CONCLUSIONS}

In this paper, a method for robust fault detection based on interval observation using zonotopes considering the effect of unknown inputs is presented. This approach use interval models to describe parameter uncertainty and assume a bounded description of process and measurement noise. The method presented in this paper computes the worst-case estimation for each state variable considering the effect of parameter uncertainty and noise. The method was successfully tested in an application example based on simplified longitudinal flight control system.

\section{ACKNOWLEDGEMENTS}

This work is supported by the Research Commission of the Generalitat of Catalunya (Grup SAC ref. 2005SGR00537). The work was financed as a research project with the science funds for years 2007-2010.

\section{REFERENCES}

T. Alamo, J.M. Bravo, and E.F. Camacho. Guaranteed state estimation by zonotopes. Automatica, 41(6):1035-1043, 2005.

J. Chen and R.J. Patton. Optimal filtering and robust fault diagnosis of stochastic systems with unknown disturbances. Control Theory and Applications, IEE Proceedings, 143 (1): $31-36,1996$.

J. Chen and R.J. Patton. Robust Model-Based Fault Diagnosis for Dynamic Systems. Kluwer Academic Publishers, 1999.

C. Combastel. A state bounding observer based on zonotopes. In European Control Conference (ECC'O3), Cambridge, UK, 2003.

J. Gertler. Fault Detection and Diagnosis in Engineering Systems. Marcel Dekker, 1998.

S. Hui and S.H. Zak. Observer design for systems with unknown inputs. Int. J. of Applied Mathematics and Comput. Science, 15(4):431-446, 2005.

J.Y. Keller. Fault isolation filter design for linear stochastic systems. Automatica, 35(10):1701-1706, 1999.

W. Kühn. Rigorously computed orbits of dynamical systems vithout the wrapping effect. Computing, 61(1), 1998.

D. Maksarov and J.P. Norton. State bounding with ellipsoidal set description of the uncertainty. International Journal of Control, 65 (5):847 - 866, 1996.

M. Milanese, J. Norton, H. Piet-Lahanier, and E. Walter, editors. Bounding Approaches to System Identification. Plenum Press, 1996.

R.E. Moore. Interval analysis. Prentice Hall, 1966.

V. Puig, J. Quevedo, and T. Escobet. Robust fault detection approaches using interval models. In IFAC World Congress (b'00), Barcelona, Spain, 2002.

B. Rinner and U. U. Weiss. Online monitoring by dynamically refining imprecise models. IEEE Transactions on Systems, Man and Cybernetics: Part B, 34 (4):1811 - 1822, 2004.

A. Vicino and G. Zappa. Sequential approximation of feasible parameter sets for identification with set membership uncertainty. IEEE Transactions on Automatic Control, 41:774$785,1996$.

M. Witczak. Modelling and estimation strategies for fault diagnosis of non-linear systems : from analytical to soft computing approaches, ISBN: 3-540-71114-7. Lecture Notes in Control and Information Sciences, 354. Springer-Verlag, Berlin, 2007. 